\title{
A Hybrid-Electric Driveline for Agricultural Tractors Based on an e-CVT Power-Split Transmission ${ }^{+}$
}

\author{
Claudio Rossi ${ }^{1}$, Davide Pontara ${ }^{2}$, Carlo Falcomer ${ }^{3}$, Marco Bertoldi $^{1}$ and Riccardo Mandrioli ${ }^{1, *(D)}$ \\ 1 Department of Electrical, Electronic, and Information Engineering, University of Bologna, \\ 40136 Bologna, Italy; claudio.rossi@unibo.it (C.R.); m.bertoldi@unibo.it (M.B.) \\ 2 Department of Industrial Engineering, University of Bologna, 40136 Bologna, Italy; davide.pontara@unibo.it \\ 3 Department of Computer Science and Engineering, University of Bologna, 40136 Bologna, Italy; \\ carlo.falcomer@unibo.it \\ * Correspondence: riccardo.mandrioli4@unibo.it; Tel.: +39-051-20-93589 \\ + Post-Conference Version of the Paper: e-CVT Power Split Transmission for Off-Road Hybrid-Electric Vehicles. \\ In Proceedings of the 2014 IEEE Vehicle Power and Propulsion Conference (VPPC), Coimbra, Portugal, \\ 27-30 October 2014; pp. 1-6.
}

check for updates

Citation: Rossi, C.; Pontara, D.; Falcomer, C.; Bertoldi, M.;

Mandrioli, R. A Hybrid-Electric Driveline for Agricultural Tractors Based on an e-CVT Power-Split Transmission. Energies 2021, 14, 6912. https://doi.org/10.3390/en14216912

Academic Editor: Federico Barrero

Received: 30 August 2021

Accepted: 19 October 2021

Published: 21 October 2021

Publisher's Note: MDPI stays neutral with regard to jurisdictional claims in published maps and institutional affiliations.

Copyright: (c) 2021 by the authors. Licensee MDPI, Basel, Switzerland. This article is an open access article distributed under the terms and conditions of the Creative Commons Attribution (CC BY) license (https:/ / creativecommons.org/licenses/by/ $4.0 /)$.

\begin{abstract}
This paper proposes a full-hybrid driveline based on an electric continuously variable transmission (e-CVT), which is inspired by the car industry's most successful solution. The paper describes the operating principle, the system architecture, and the control scheme of the proposed driveline. An analysis of four possible operating modes shows that the e-CVT driveline leads to a performance similar to that of conventional tractors, as well as unusual features such as power boost, full-electric mode, optimized auxiliary drive and electric power delivery capability. The compact layout proposed for the e-CVT also makes it possible to simplify the overall layout of the tractor, particularly during the installation of both the thermal engine and the cooling system.
\end{abstract}

Keywords: power-split; hybrid powertrain; hybrid driveline; epicyclical gear set; tractors

\section{Introduction}

The electric continuously variable transmission (e-CVT) system is the most widely used full-hybrid-electric drive system configuration in automobiles. This solution, introduced in 1997 [1], has been largely demonstrated to have the functional requirements, performance, efficiency, and manufacturing complexity to be compatible with the automotive sector. In summary, the characteristics of a full-hybrid e-CVT transmission for cars are as follows:

- It makes the ratio between engine speed and wheel speed continuously variable over a very wide range.

- $\quad$ Enables pure electric driving for a reasonable range.

- Reduces fuel consumption and polluting emissions by at least $40 \%$ compared with traditional vehicles (internal combustion engines (ICEs) and mechanical gearboxes in urban driving), based on the Worldwide Harmonized Light-Duty Vehicles Test Procedure (WLTP) [2].

- It inherently implements several auxiliary functions that are normally accomplished through the combined use of the gearbox, clutch, and starter-generator [3,4].

With regard to industrial vehicles, particularly agricultural tractors, several operating conditions are noticeably similar to driving a car in urban traffic [5]. Moving at zero speed or very low speed for a long time (e.g., harvesting, driving tools at a standstill) or repeated accelerations and decelerations in the low-speed range (e.g., continuous maneuvering in limited space) are two of the possible examples.

Furthermore, in various agricultural applications, the full-electric operation (EV mode) of a tractor could be of great interest for the following reasons: 
- The use of zero-emission tractors in closed environments (e.g., greenhouses and stables) improves the air quality, making the workplace healthier for the farmers and increasing the quality of the food produced [6].

- Electric energy can be obtained from renewable sources, especially from local renewable electric energy production sites; hence, a fully fuel-free food production chain can be achieved [7].

Compared to automobiles, a small- to medium-sized tractors must be characterized by very high tractive effort capabilities at low speeds for typical low-speed/high-load operations (e.g., plowing), while being able to provide high power at low-medium speeds for operating soil tillage implements that require both pulling and power supply (e.g., seeders), and at high vehicle speeds (e.g., for towing trailers on-road). These heavy-duty operating conditions are typically applied for many hours.

A significant difference between tractor and car powertrains lies in the need for the power take-off (PTO) additional shaft that is used for powering agricultural implements. The PTO requires speed control that is independent from the wheels' speed, and it is considered a critical matter in the design of modern tractors [8].

By combining the characteristics mentioned above, the minimum list of functionalities a tractor must fulfill are as follows:

- To enable continuously variable ratio between engine and wheel speed over a very wide range of ground speeds.

- To enable PTO speed control, independent from the wheels' speed control.

- To allow adjustment of the ICE operating point for enhanced fuel economy and reduction of pollutant emissions.

- To allow "combustion-only mode", where the driveline is powered solely by the combustion engine, with one or more fixed ratios between engine and wheels; here, electric motors are deactivated.

- To allow "electric-only mode". The electrical system operates both the traction and the PTO using the energy stored in a battery pack; here, the combustion engine is off.

- To power ancillaries and external hydraulic loads with optimum energy saved.

- To supply external electric loads (e.g., electric implements and tools) that are connected to dedicated sockets on the tractor.

In addition to the above functionalities and requirements, the hybridization of the driveline of agricultural tractors should enable the downsizing of the thermal engine and enable them to meet the new non-road emissions (NRE) regulations (EU-STAGEV, EPA-TIER4, China IV) [9].

The requirements listed above can be satisfied with a plurality of configurations of full-hybrid powertrains, which can be classified as the series type, the parallel type, the power-split type, and their combinations [10]

The reference configuration of a series type transmission [11] is based on the combustion engine's direct connection with a variable speed electrical generator. In this configuration, the power used for traction, PTO, and auxiliaries entirely comes from a double electro-mechanical conversion. Interesting features of the series driveline are the possibility of efficiently supplying tools via an electric socket (electric PTO) [10], the absence of mechanical coupling between the engine and the wheels [12], and the power splitting over multiple drives. The introduction of a battery pack allows peak shaving capability, opens to the possibility of downsizing the ICE, and allows a quicker power supply response, cutting the lag due to generator transients [13]. The complexity and large sizing of the electric generation unit and all the electric motors prevent the possibility of finding a transmission layout solution based on the series configuration that can be economically installed in a small- or medium-sized tractor.

Several manufacturers are presently relying on parallel hybrid powertrains. In the parallel type, one electrical machine-operating either as a motor or generator-is usually integrated into a traditional driveline between the engine and the mechanical gearbox, in the so-called positions P1 and P2 as described in [14,15]. With this configuration, the 
electrical machine assists the combustion engine during transients, boosting the maximum available power to the driveline. Using a single electrical machine in the parallel type represents a trade-off between system performance and complexity, while providing a moderate contribution to engine downsizing and fuel saving; however, it still requires the traditional drivetrain with a mechanical gearbox. This configuration opens to further upgrades of the hybrid system; for example, a considerable improvement of the engine performance can be obtained by integrating an electrical machine in the engine's turbocharger shaft [16]. More generally, improvements of parallel hybrid configurations are represented by combinations of the main motor/generator with more electrical machines coupled to wheels, auxiliary drives, and the PTO [17]. The power fractioning, utilizing several electric drives, leads to better use of the electrical energy, improves the fuel efficiency, and opens to the possibility of having tractors that are capable of operating in the full-electric mode for some types of mission. As a drawback, this approach increases system complexity and system cost.

Besides the main two families of hybrid driveline (the series type and the parallel type) the power-split solution is an alternative approach that tries to merge the advantages of the other approaches. A power-split hybrid configuration was introduced in 1935 [18] as an electric variable transmission (EVT), but the most important configuration-which has had the most significant diffusion in the automotive field - is the one called electric continuously variable transmission (e-CVT) [19]. Several configurations of hybrid drivelines based on the power-split e-CVT have been presented, and two main categories can be recognized, encompassing most of the proposed solutions: the input split and the compound split [20]. Compound power-split transmissions [21-26] require two or more epicyclical gear sets and allow a substantial variation in the engine-wheel speed ratio. This solution, that was developed for high-performance cars, appears to be too complex and expensive to be used on small agricultural tractors.

The input power-split e-CVT driveline is considered of interest for tractor application due to its undisputed success in the hybrid car sector. The expected functionalities and performance are achieved, despite the reduced sizing of the electro-mechanical conversion systems [27]. The most promising solution for tractor application is the most straightforward e-CVT power-split transmission configuration, which comprises a planetary gear set and two electric machines, installed between the thermal engine and the differential gearbox. tab 1 represents the basic configuration of this e-CVT in the configuration adopted by a full-hybrid driveline for passenger cars. In this configuration, the planetary gear set divides the power entering from the thermal engine, connected to the carrier gear, between the "direct path" and the "converted path". The "direct path" transfers power from the engine to the ring, and then to the final drive and the wheels, using mechanical gearings. The "converted path" transfers power through two electric machines: MG/1 connected to the sun gear and MG/2 connected to the ring. Both machines transfer electric power to the same DC bus through their power electronics, where a battery pack stores a limited amount of electric energy $[1,20,27,28]$.

In this paper, the solution presented for the tractor powertrain is founded on the consolidated e-CVT power-split architecture given in Figure 1, and this solution is adapted to tractor application, as proposed for the first time in [29]. This paper extends the description of the basic principles of applying the e-CVT to the tractor, proposes a design criterion that provides detailed data of all the subsystems, describes a reference control scheme for hybrid mode, and analyzes the behavior of the driveline in the hybrid mode under the various typical operating conditions that can occur for an agricultural tractor. In order to numerically validate the performance of the proposed solution, a model of the main components of the powertrain was created. A basic control system was developed, and the most severe operating conditions for a vehicle of this type were simulated. The numerical tests include the combined control of wheel speed and PTO speed under different transient conditions. The results show that the proposed powertrain architecture can perform all the functions of a tractor driveline and reach performances comparable to those of fully thermal vehicles equipped with modern automatic transmission (either a hydraulic torque 
converter or CVT). A full-scale tractor prototype, based on the proposed layout solution, was also realized. From this layout, it is clear that the proposed transmission provides the possibility of introducing important changes to the layout of a small multipurpose tractor, that could improve the use of space on board. Furthermore, it is shown in this paper that the power-split e-CVT transmission proposed for the tractor driveline takes advantage of some inherent features of the e-CVT that are not currently used in car applications, such as the potential for powering external electrical loads and the optimal speed control of auxiliary loads in EV mode. The prototype will allow, in the future, experimental validation of the numerical results covered by this manuscript, as well as offering a solid base for developing advanced control algorithms and energy management systems.

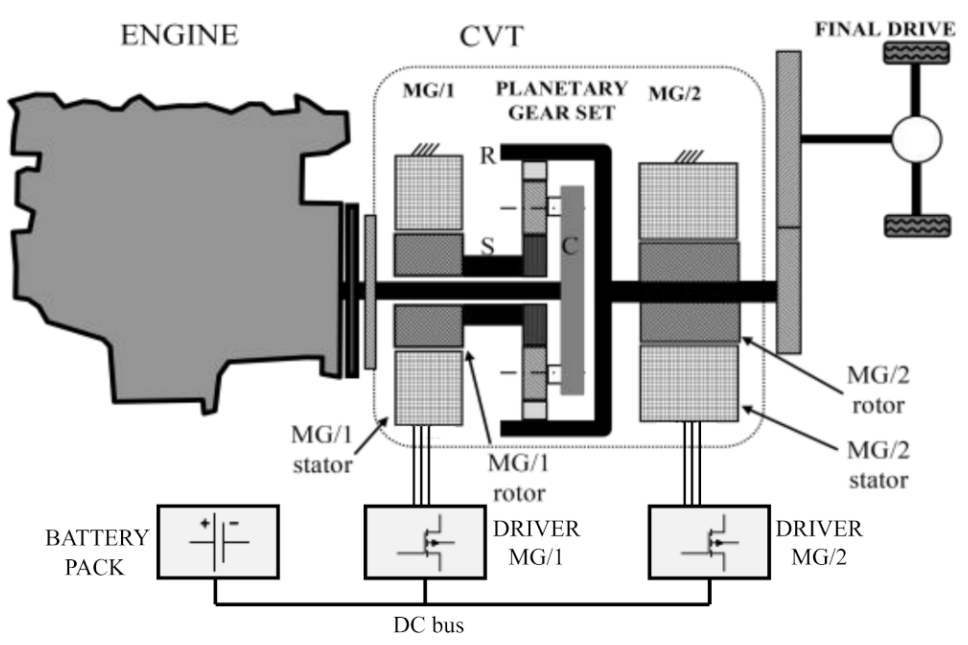

Figure 1. Basic configuration of a vehicular driveline based on the power-split e-CVT.

This paper is organized as follows. Section 2 recalls the fundamental equations of the epicyclical gear set, giving the fundamental relations that guide either the sizing of the driveline, or the setting up and tuning of the control strategy. Section 3 presents the vehicle data and introduces the transmission scheme with all the related data. This section also describes all four possible operating modes of the transmission. Section 4 focuses on the e-CVT control scheme with particular attention on the full-hybrid mode. Section 5 presents all the simulation results to demonstrate the functionality and performance of the proposed hybrid mode. Section 6 shows the experimental vehicle resulting from this work. Section 7 concludes the manuscript and opens the way to subsequent analyses that are only hinted at in this article, such as the evaluation of the energy performance of the entire vehicle, the optimal sizing of the battery, and the design of the individual parts of the electro-mechanical conversion system.

\section{Epicyclical Gear Set}

In the following subsection, the relevant epicyclical gear set kinematic, static, and dynamic equations are recalled.

\subsection{Kinematic and Static Equations}

In Figure 1, the epicyclical (planetary) gear set scheme, at the base of the e-CVT, is displayed.

The planetary gear set's quasi-static behavior can be evaluated through fundamental Equation (1), known as the Willis equation, which describes the speed relationship of the three main components. In this assessment, the epicyclical gear set is considered as an 
ordinary gear train, mounted on a rotating frame, being the latter integral with the carrier. Therefore, the ring-sun relative speed ratio can be written as follows:

$$
\tau_{0}=\frac{\omega_{S}-\omega_{C}}{\omega_{R}-\omega_{C}}
$$

where $\omega_{R}, \omega_{S}$, and $\omega_{C}$, are the speed of the ring $(R)$, the sun $(S)$, and the carrier $(C)$, respectively, while $\tau_{0}$ represents the epicyclical gear ratio, which is defined as follows:

$$
\tau_{0}=-\frac{N_{R}}{N_{S}}
$$

where $N_{S}$ and $N_{R}$ are the sun and ring teeth numbers, respectively.

According to the Willis equation (Equation (1)), the speed ratio between two gear set elements is defined by the third element's speed.

The following notation is defined and used in the paper. Electrical machines' power is defined to be positive if they operate as motors. In contrast, the power of CVT elements is positive if power is delivered to the epicyclical gear set (power entering in the gear set). Ring and MG/2 speeds are defined as positive if they cause the vehicle to move in a forward direction. $M G / 1$ positive speed corresponds to the rotating direction of $M G / 1$ if the vehicle is stopped $\left(\omega_{R}=0\right)$ and the ICE is running.

By applying the power balance equation to the gear from the Willis formula, the two following fundamental equations of the torque are obtained:

$$
\begin{gathered}
T_{C}+T_{R}+T_{S}=0 \\
\frac{T_{R}}{T_{S}}=-\tau_{0}
\end{gathered}
$$

where $T_{R}, T_{S}$, and $T_{C}$, are the torques applied to the ring $(R)$, the sun $(S)$, and the carrier (C), respectively.

Equations (3) and (4) show that the third shaft torque defines the amount of torque that can be transferred between two planetary gear set shafts. Indicating the carrier shaft torque with $T_{C}$, the sun and ring torques are set by the gear ratio $\tau_{0}$ as follows:

$$
\begin{gathered}
T_{R}=\tau_{0} \frac{T_{C}}{1-\tau_{0}} \\
T_{S}=\frac{T_{C}}{1-\tau_{0}}
\end{gathered}
$$

Defining one of the gear set elements as the controller, the speed ratio analysis becomes straightforward. From Equation (1), the carrier $(C)$ speed $\omega_{C}$ is obtained from the ring $(R)$ speed $\omega_{R}$ as a function of the sun $(S)$ speed $\omega_{S}$, which acts as the following regulation element:

$$
\omega_{C}=-\frac{\tau_{0}}{1-\tau_{0}} \omega_{R}+\frac{1}{1-\tau_{0}} \omega_{S}
$$

According to Equation (7) and the scheme of Figure 1, the gear ratio regulation between the wheels (coupled to the ring) and the IC engine (coupled to the carrier) can be achieved by varying the sun speed (coupled to an electric machine). From Equation (7), for any ring speed $\omega_{R}$, it is possible to find a carrier speed, referred to as base carrier speed $\omega_{\mathrm{C} 0}$ (Equation (8)) at which the sun is stationary $\left(\omega_{S}=0\right)$, as follows:

$$
\omega_{C 0}=-\frac{\tau_{0}}{1-\tau_{0}} \omega_{R}
$$


Introducing $\omega_{C 0}$ from kinematic Equation (8) in $P_{R}=T_{R} \omega_{R}$, the ring and sun powers become proportional to the carrier power as follows:

$$
\begin{gathered}
P_{R}=\frac{\omega_{C 0}}{\omega_{C}} P_{C} \\
P_{S}=\left(\frac{\omega_{C 0}}{\omega_{C}}-1\right) P_{C}
\end{gathered}
$$

By analyzing Equations (9) and (10), three operating modes of the gear set, depending on the sun speed $\omega_{S}$, are recognized as follows:

1. Mode 1: $\omega_{C}=\omega_{c 0}$, in this condition, the sun speed $\omega_{S}$ is zero. As stated in Equation (6), by applying reaction torque at the sun, torque transferring between the carrier and the ring can be achieved. In this condition, all the power provided by the ICE entering the carrier is transferred to the wheels through the ring, $P_{C}=P_{R}$, as the sun power is null.

2. Mode 2: $\omega_{C}>\omega_{c 0}$, the sun rotates in a positive direction (positive sun speed $\omega_{S}$ ), and power is supplied by the sun $\left(P_{S}<0\right)$. In this mode, the carrier input power produced by the ICE is higher than the ring output power transferred to the wheels: $P_{R}<P_{C}$. In other words, a fraction of the power produced by the ICE is transferred to the electrical machine, which behaves as a generator.

3. Mode 3: $\omega_{C}<\omega_{c 0}$, the sun speed $\omega_{S}$ is negative, and power is entering in the sun: $\left(P_{S}>0\right)$. Therefore, the carrier input power produced by the ICE is lower than the ring output power transferred to the wheels: $P_{R}>P_{C}$. The electric machine acts as a motor providing power.

Depending on whether $\omega_{C 0}$ is higher or lower than the engine (carrier) speed, the electrical machine (connected to the sun), which regulates the speed ratio to the wheels, may act as a generator or a motor, respectively.

\subsection{Dynamic Equations}

The planetary gearbox dynamic equations can be obtained by applying the torque balance equation $T_{\text {mot }}-T_{\text {load }}=J \dot{\omega}$ to torque Equations (5) and (6).

For instance, the counteraction-a product of torque transferring between carrier and ring-causes an acceleration of both carrier and ring.

Thus, the ring dynamic equation is as follows:

$$
-\frac{\tau_{0}}{1-\tau_{0}} T_{C}+T_{R}=J_{R} \dot{\omega}_{R}-\frac{\tau_{0}}{1-\tau_{0}} J_{C} \dot{\omega}_{C}
$$

where $J_{R}$ and $J_{C}$ are the ring- and carrier-connected rotating masses' moments of inertia, respectively.

Similarly for the sun as follows:

$$
\frac{1}{1-\tau_{0}} T_{C}+T_{S}=J_{S} \dot{\omega}_{S}+\frac{1}{1-\tau_{0}} J_{C} \dot{\omega}_{C}
$$

where $J_{S}$ is the sun-connected rotating mass' moment of inertia.

By replacing Equation (7) into Equations (11) and (12), it can be obtained that:

$$
\begin{gathered}
-\frac{\tau_{0}}{1-\tau_{0}} T_{C}+T_{R}=\left[J_{R}+\left(\frac{\tau_{0}}{1-\tau_{0}}\right)^{2} J_{C}\right] \dot{\omega}_{R}-\frac{\tau_{0}}{\left(1-\tau_{0}\right)^{2}} J_{C} \dot{\omega}_{S} \\
\frac{1}{1-\tau_{0}} T_{C}+T_{S}=\left[J_{S}+\left(\frac{1}{1-\tau_{0}}\right)^{2} J_{C}\right] \dot{\omega}_{S}-\frac{\tau_{0}}{\left(1-\tau_{0}\right)^{2}} J_{C} \dot{\omega}_{R}
\end{gathered}
$$


Equations (7), (13) and (14), rearranged as follows:

$$
\omega_{S}=\tau_{0} \omega_{R}+\left(1-\tau_{0}\right) \omega_{C},
$$

give a complete set of equations useful for the planetary gear set dynamic behavior description.

The transmission state-space model can be obtained from Equations (12)-(15) as follows:

$$
\begin{aligned}
\dot{\mathbf{x}}(t) & =\mathbf{A} \mathbf{x}(t)+\mathbf{B} \mathbf{u}(t) \\
\mathbf{Y}(t) & =\mathbf{C} \mathbf{x}(t)+\mathbf{D} \mathbf{u}(t)
\end{aligned}
$$

where vectors $\mathbf{x}(t), \mathbf{u}(t)$, and $\mathbf{Y}(t)$ are the state, the input, and the output, respectively. The latter set of vectors is defined as follows:

$$
\begin{aligned}
& \mathbf{x}=\left[\begin{array}{ll}
\omega_{R} & \omega_{S}
\end{array}\right]^{T} \\
& \mathbf{Y}=\left[\begin{array}{lll}
\omega_{R} & \omega_{S} & \omega_{C}
\end{array}\right]^{T} \\
& \mathbf{u}=\left[\begin{array}{lll}
T_{R} & T_{S} & T_{C}
\end{array}\right]^{T}
\end{aligned}
$$

Equation (16) state-space matrices are as follows:

$$
\begin{aligned}
& \mathbf{A}=0 \\
& \mathbf{B}=\frac{1}{h}\left[\begin{array}{lll}
\left(1-\tau_{0}\right)^{2} J_{S}+J_{C} & \tau_{0} J_{C} & -\left(1-\tau_{0}\right) \tau_{0} J_{S} \\
\tau_{0} J_{C} & \left(1-\tau_{0}\right)^{2} J_{A}+\tau_{0}^{2} J_{C} & \left(1-\tau_{0}\right) J_{S}
\end{array}\right] \\
& \mathbf{C}=\left[\begin{array}{cc}
1 & 0 \\
0 & 1 \\
-\frac{\tau_{0}}{1-\tau_{0}} & \frac{1}{1-\tau_{0}}
\end{array}\right] \\
& \mathbf{D}=0
\end{aligned}
$$

where:

$$
h=\left(J_{A}+\tau_{0}^{2} J_{S}\right) J_{C}+\left(1-\tau_{0}\right)^{2} J_{A} J_{S}
$$

This state-space model of the e-CVT constitutes the core of the transmission control scheme that is described in Section 3.

\section{Transmission Layout}

In order to correctly size the proposed driveline for the specific vehicle (tractor), with all the expected features described in Section 1, the design criteria given in [30] are applied. The inputs for this method are the torque-speed characteristics of the given combustion engine, the absolute maximum torque-speed characteristics requested at the wheels, and a primary control strategy of the combustion engine rotating speed. Using this method, the epicyclical gear ratio, driveline gear ratios, and the absolute torque-speed limit characteristic demanded at the two electrical machines are determined [31].

The main physical specifications of the considered tractor are given in Table A1 (Appendix A). The key data of the proposed diesel ICE are gathered in Table A2 (Appendix A). The selected gear ratios and geometrical data of the proposed transmission are given in Table A3 (Appendix A). The main data of the two machines embedded in the power-split e-CVT assembly are contained in Table A4 (Appendix A).

Figure 2 shows the basic layout scheme of the proposed hybrid-electric driveline. This scheme represents the power-split e-CVT arrangement, based on the configuration of two coaxial and concentric electrical machines, strictly integrated with the epicyclical gear set. 


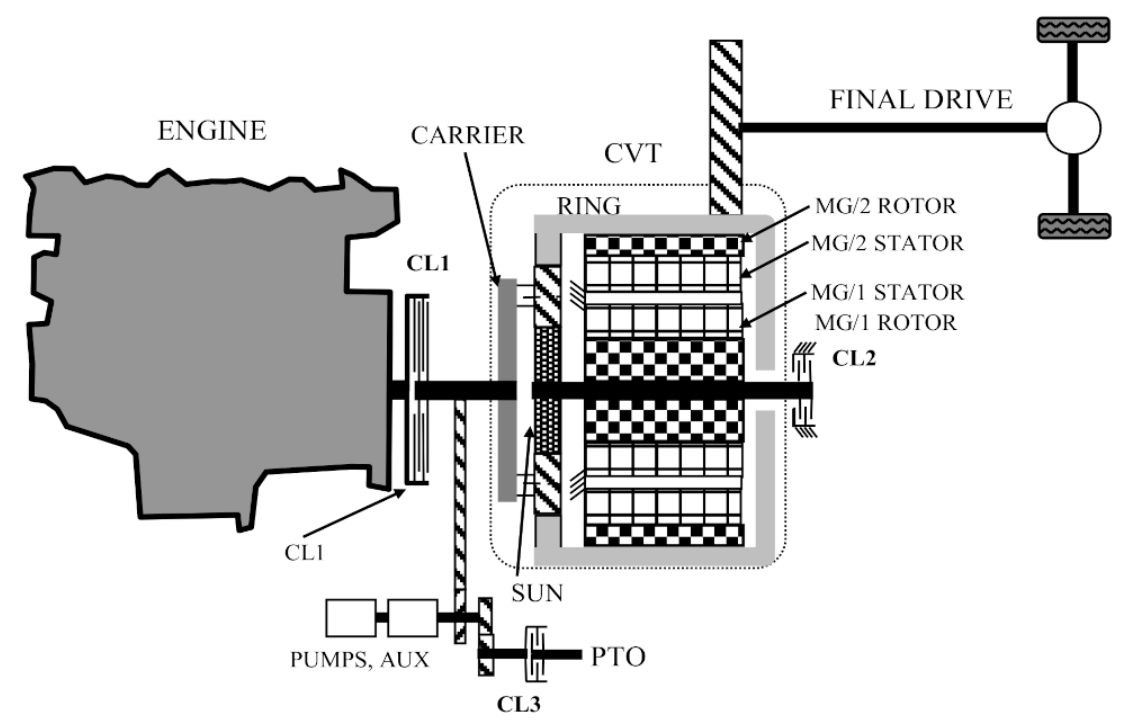

Figure 2. Layout of the concentric coaxial power-split e-CVT, integrated into a tractor's transmission.

In the scheme of Figure 2, the planetary gear set elements are coupled, following the solution described in Section 1. The following features emerge:

- The carrier is connected to the combustion engine, auxiliary loads, and the PTO.

- The MG/2 electric machine is keyed to the Ring gear, and these are connected to the final drive.

- $\quad$ The sun ring is coupled with the MG/1 electric machine only.

For enabling the operation of the powertrain in all the possible modes, three electroactuated clutches are required as follows (as it can be observed in Figure 2):

- CL1: connects the ICE crankshaft to the carrier shaft.

- CL2: can lock the sun in a stopped position, as its driven shaft is connected to the frame.

- CL3: activates the PTO by coupling it to the carrier shaft.

The essential scheme of Figure 2 does not include the final drives, which include further gearboxes and clutches for managing the following:

- Reverse gear.

- $\quad$ All-wheel drive (AWD).

- Parking braking system.

- Neutral gear.

This design is based on a final drive, which is the standard used on commercially available tractors and will not be discussed throughout this paper.

Figure 3 gives the torque and power characteristics of the chosen diesel combustion engine. As can be seen in the figure, the output characteristics are unusual for industrial engines used on tractors, as the maximum torque region is quite narrow and is provided at relatively high speeds, if compared with typical industrial engines. In fact, the chosen diesel ICE was initially designed for car applications. This engine is characterized by higher rotating speeds, lower emissions, and lower costs than the diesel engines that are traditionally employed in tractors. The lack of a broad maximum torque region is not a concern, thanks to the hybrid transmission. 


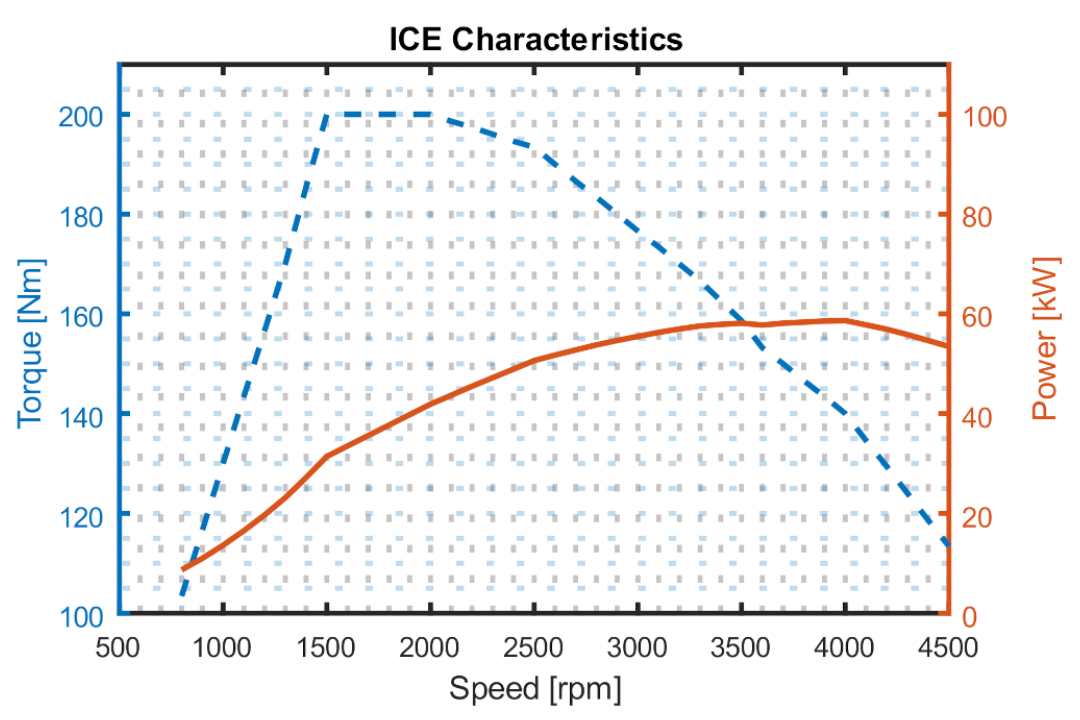

Figure 3. Internal combustion engine maximum torque and power characteristics.

The possible operating modes of the transmission can be easily associated with the configuration of the clutches that are represented in Figure 2 and have been described. Five basic operating modes can be defined for this transmission. A basic description of all operating modes is given in the following paragraph, while a detailed description of full-hybrid mode is given in Section 4.

It is worth noting that additional electric loads can be added to the main DC-link, which is sustained by the battery pack, in any operating condition. In the case of the hybrid mode, the control system regulates the power flow from the engine to operate the electric load without discharging the battery, preserving the regulation of either the ground speed or the PTO speed.

\subsection{EV Mode}

The combustion engine (ICE) is switched off and decoupled from the transmission by opening CL1. MG/2 solely controls traction by using power from the battery pack. The operation of the vehicle is limited to the low-medium speed range. A high tractive effort can be produced, even if the maximum power output is mainly limited by the power capability of the battery pack.

If the PTO is not used then clutch CL3 is open, and the PTO is disconnected from the carrier driveline. The e-CVT is used to regulate the auxiliaries' speed (hydraulic pumps) and is permanently connected to the carrier driveline. CL2 is always open and MG/1 is speed-regulated-according to the Willis equation-to maintain the carrier's target speed, regardless of the wheel speed. In this way, it is possible to drive the auxiliaries at the optimum efficiency operating point for any required auxiliary power. This feature prevents unnecessary losses in the auxiliary hydraulic circuits, reducing power requests from the batteries and increasing the EV mode range.

If the PTO is used then clutch CL3 is closed, and PTO is connected to the carrier driveline. MG/1 speed is controlled to drive the PTO at the requested speed (typically $540 \mathrm{rpm}$ or $1000 \mathrm{rpm}$ ), regardless of the wheels' speed. Mechanical auxiliaries are driven at a constant speed; although, in this condition, the PTO speed has priority and, therefore, the maximum efficiency of the hydraulic circuit might not be reached.

\subsection{Full-Hybrid Mode}

In this operating condition, the ICE is running and connected to the driveline by closing the clutch CL1. CL2 is open. The main hybrid-electric functionality is obtained through the control of MG/1, MG/2, and ICE. The control of wheel and engine speed is combined with power flow management, following the principle described in Section 4. 
The speed of the auxiliary drives is determined by the engine (carrier) speed, provided that the reference speed of the engine is compliant with the pumps' speed constraints from the hydraulic circuit. The PTO can be engaged with the same ICE-carrier shaft by closing CL3. Whist the PTO is operational, the master reference speed for the engine is determined by the constant PTO reference (540 rpm or $1000 \mathrm{rpm}$ ); consequently, the auxiliary pumps' speed is fixed.

\subsection{Combustion-Only Mode}

Similarly to the full-hybrid mode, the ICE is operational and connected to the driveline by closing CL1; although, CL2 is closed, and hence, MG/1 is stopped. Therefore, the main hybrid electric functionalities are disabled. The sun reaction torque is produced directly by the CL2 clutch. As described in Section 2, in this condition, an element of the epicyclical gear set is locked (the sun), so the gear set performs as an ordinary gear, providing a defined gear ratio between the engine and wheels. In other words, the wheels' speed is uniquely determined by the ICE rotating speed; consequently, the MG/2 driver can be switched off, as the ICE provides the full traction power. Still, MG/2 will be free-spinning, as it is built-in with the final drive. Auxiliary drives' (and the PTO if CL3 is closed) speeds are directly driven by the engine speed. Speed decoupling between wheels and auxiliaries-PTO is not possible in this mode.

The full-combustion mode can be selected at the medium-high speed of the tractor.

\subsection{Parallel-Hybrid Mode}

Parallel-hybrid mode is based on the combustion-only mode configuration. The additional feature is given by the possibility of also powering MG/2. In this way, the electro-mechanical conversion on MG/2 can either add power to, or subtract power from, the final driveline. Two main goals of this operating mode are as follows:

- $\quad$ Positive or negative high torque can be applied for a short time, either for enhancing vehicle acceleration performance or for regenerative braking.

- A low value of generating torque can be applied for a longer time to recharge the battery.

\subsection{Cranking}

Cranking is a transient mode (lasting about $2 \mathrm{~s}$ ) that allows switching from EV mode to hybrid mode without additional hardware coupled to the engine.

Starting from EV mode with a low carrier speed, CL1 is closed, and MG/1 is speed regulated to produce the proper speed ramp on the carrier for cranking the engine. MG/2 is demanded to integrate the torque, subtracted by the cranking during this transient, to keep the wheel speed undisturbed.

\section{Design and Control of the e-CVT}

This section presents a discussion of the control and regulation of the proposed e-CVT, as operated in full-hybrid mode.

\subsection{Full-Hybrid Mode Basic Control Strategy}

In this paragraph, the essential control strategy of the powertrain is described, concerning steady-state conditions. Following the simple strategy already introduced in [30] and [29], the basic reference speed $\omega_{I C E}^{*}$ for the ICE is directly imposed by the ground speed, following these criteria:

- $\quad$ For backward speed, vehicle stopped, and very low forward speed, the engine is kept at its idling speed of $900 \mathrm{rpm}$.

- At low speed $\left(4<v_{G N D}<7 \mathrm{~km} / \mathrm{h}\right)$, ICE linearly accelerates from $900 \mathrm{rpm}$ to $1400 \mathrm{rpm}$.

- At medium-low speed $\left(7<v_{G N D}<12 \mathrm{~km} / \mathrm{h}\right)$, engine speed is further increased up to $2000 \mathrm{rpm}$. 
- At medium-high speed $\left(12<v_{G N D}<20 \mathrm{~km} / \mathrm{h}\right)$, the engine speed is kept constant at $2000 \mathrm{rpm}$, as this is the optimal operating condition in terms of fuel efficiency and emissions of the selected engine.

- At full speed $\left(v_{G N D}>20 \mathrm{~km} / \mathrm{h}\right)$, engine speed is further increased up to $4000 \mathrm{rpm}$ because of the fixed gear ratio with the wheels.

The blue line in Figure 4 shows the reference ICE base speed $\left(\omega_{\text {ICE0 }}^{*}\right)$ vs. ground speed $\left(v_{G N D}\right)$. This speed reference is not directly applied to the engine, but it is modified by the control system in order to satisfy the actual power request, as is described in Section 4.2.

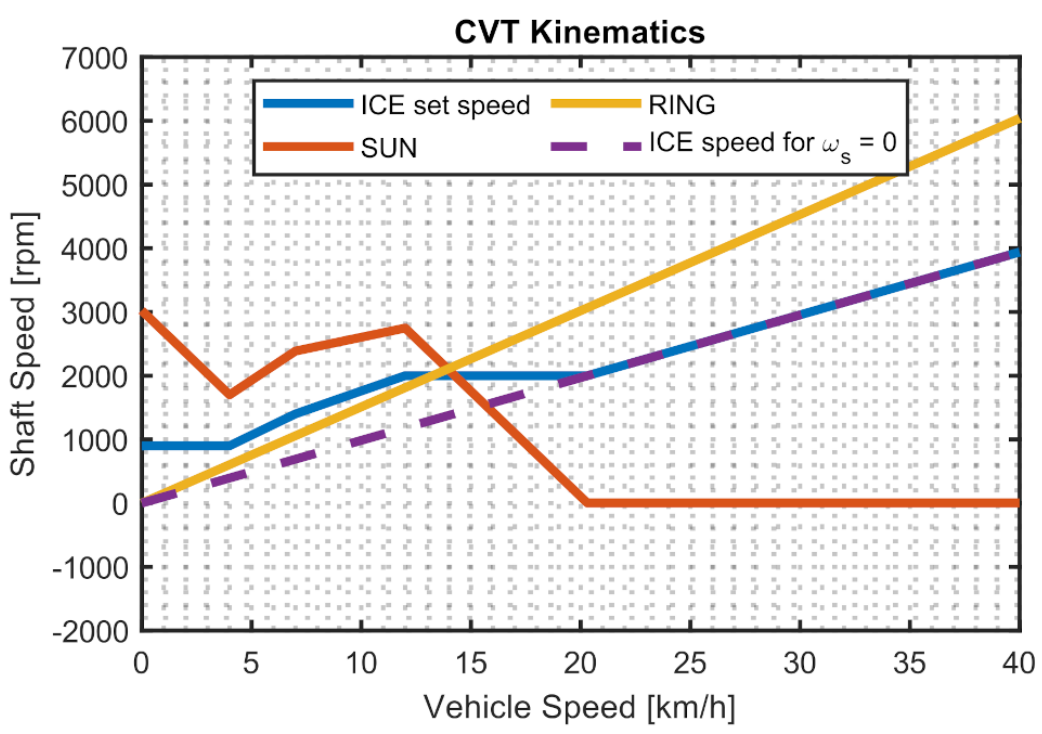

Figure 4. Kinematic relationship of the main rotating elements of the transmission.

Figure 4 also shows the speeds of the other elements of the gear set, which are the sun gear and the ring gear, which are related to the vehicle speed and the ICE speed, according to the Willis equation.

Based on the kinematic and static relationships, introduced in Section 2, this basic engine control strategy yields to select the epicyclical gear ratio $\tau_{0}$ and the other main gear ratios $\tau_{1}, \tau_{5}$, and $\tau_{6}$, which are shown in Table A3 (Appendix A). For the mapped reference ICE base speed, the resulting speeds of MG/1 (sun) and MG/2 (ring) are given in Figure 4 as a function of ground speed. This basic transmission control strategy allows the following:

- Exploitation of the engine in the region of maximum engine torque, approximately in the speed range 1200-2500 rpm, for the tractor ground speed in the range $5-15 \mathrm{~km} / \mathrm{h}$.

- Use of the MG/1 machine in generating mode. It can be verified by checking the maximum torque profile, requested to MG/ 1 and shown in Figure 5. Specifications of MG/1, shown in Table A4 (Appendix A), are obtained from this diagram.

- Use of a fixed gear ratio between the engine and final drive, at high speed, which yields the possibility of shutting down the electric drives.

The specifications of the driveline are completed with the selection of the MG/2 machine. The two following criteria define the minimum mechanical output of this machine:

- $\quad \mathrm{MG} / 2$ adds torque at low speed, delivering the gap between total demanded torque and torque available at the ring coming from the transmission.

- $\mathrm{MG} / 2$ utilizes (as a motor) the power that MG/1 generates. 

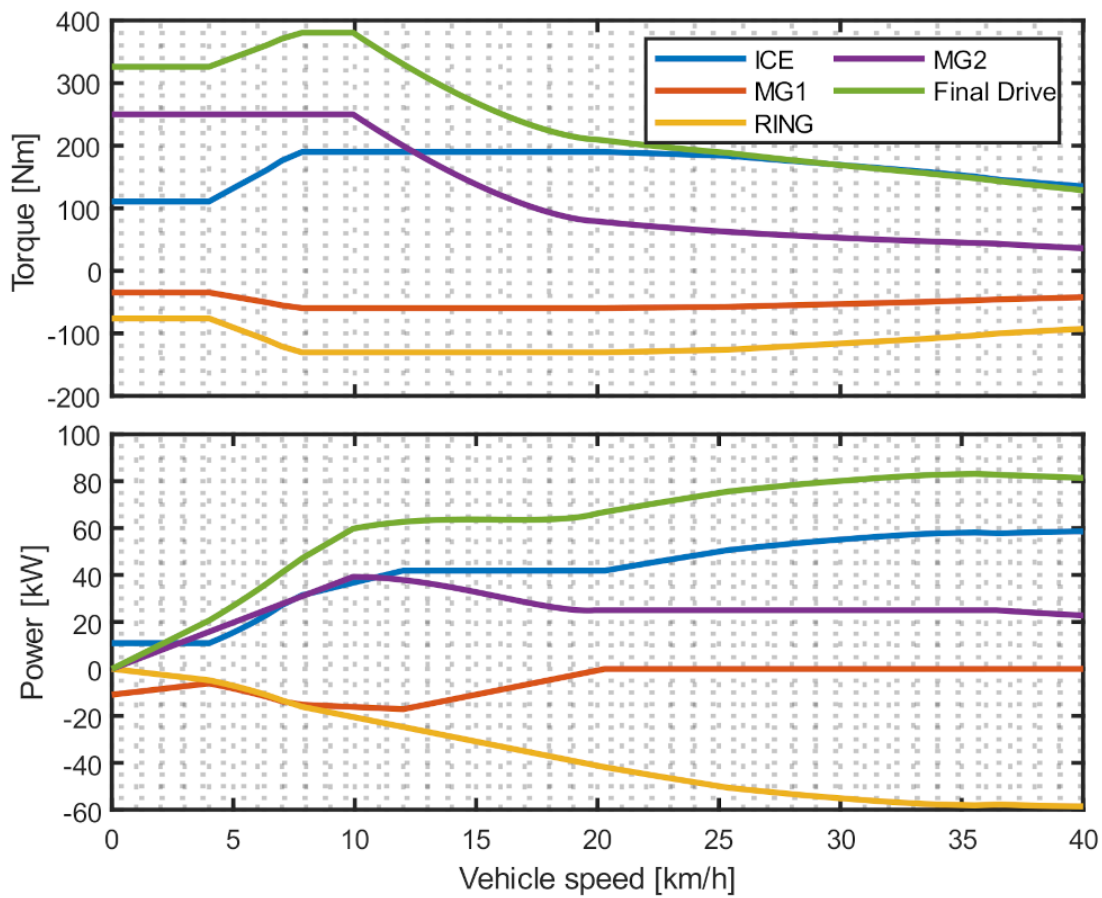

Figure 5. Maximum torque and power of the e-CVT elements.

The main specifications $\mathrm{MG} / 2$, resulting from these assumptions, are also given in Table A4 (Appendix A). The limit characteristics of both the machines, MG/1 and MG/2, are extracted from Figure 5 and represented in Figure 6.

MG1 \& MG2

Characteristics
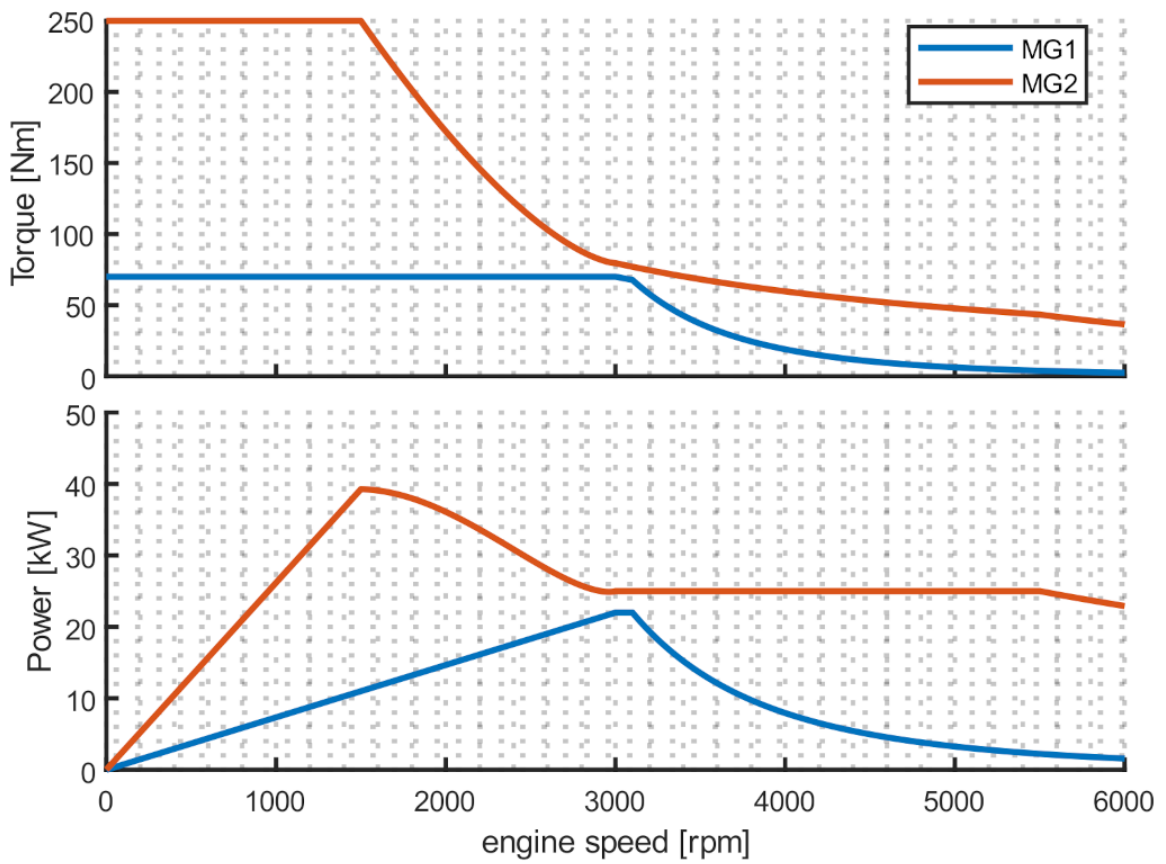

Figure 6. Maximum torque and power of the selected MG1 and MG2 drives. 
The type-selection process and the design criteria for these two machines can be found in [30]. Using this approach, the characteristics demanded for MG/1 can be obtained by a surface permanent magnet synchronous motor (SMPM-SM) with an internal rotor. This choice is also justified by the higher rotation speed that keeps the torque rating low, leading to a small machine size that can fit easily in the innermost volume of the transmission. The characteristics demanded for MG/2 can be easily obtained by using different machine configurations based on either of the synchronous configurations-as internal permanent magnet synchronous motor (IPM-SM) or permanent magnet assisted reluctance synchronous motor (PMAR-SM) — or on an induction motor (IM) configuration. The induction motor was chosen for its greater simplicity of construction in the external rotor configuration and its lower manufacturing cost. For MG/2, using a synchronous-type motor could be an interesting option for increasing the conversion efficiency and then simplifying the cooling system.

\subsection{Control Scheme}

The high-level control scheme of the e-CVT power-split transmission in full-hybrid mode is represented in Figure 7.

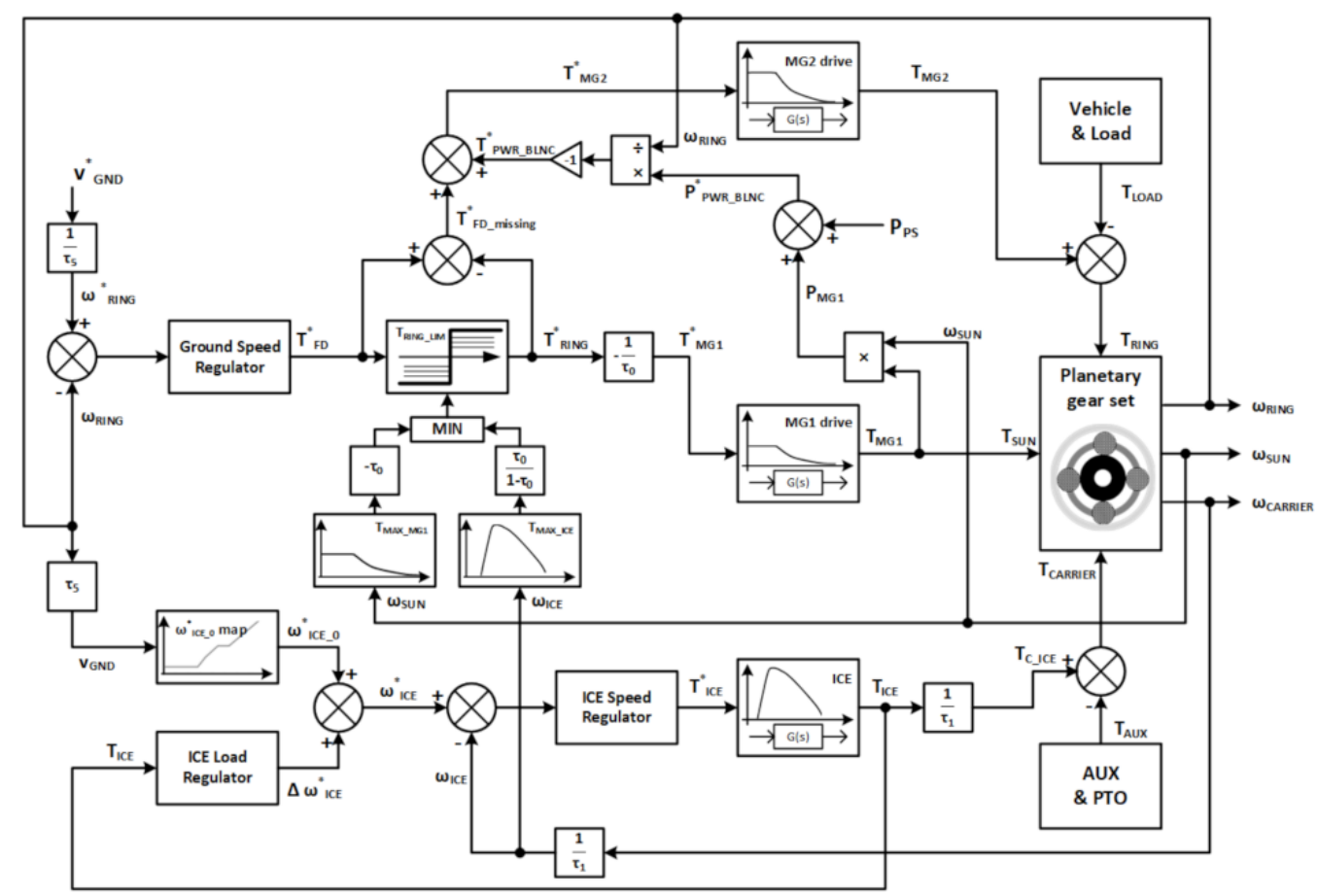

Figure 7. Control scheme of the e-CVT power-split transmission in full-hybrid mode.

The control scheme is based on the dynamic model of the planetary gear set given in Section 2, on the complete set of specifications and diagrams given in Section 2, and on the basic control principle proposed in Section 4.1. The control scheme inputs are the ground speed $V_{G N D}^{*}$, the electric power to be generated, and the requested power for PTO and hydraulic ancillaries; these provide, as outputs, the torque references for ICE, MG/1 and MG/2. For controlling the vehicle speed and the electric power in any operating condition, the control scheme can change the operating points from the pre-mapped base characteristics, given in Figure 4, for ICE, MG/1, and MG/2.

\subsubsection{Ground Speed Regulation}

The "ground speed regulator", which is implemented at the finale drive (FD) section, regulates the vehicle ground speed $\left(V_{G N D}\right)$ using a standard PI regulator. 
The torque $\left(T_{F D}^{*}\right)$, calculated by the ground speed regulator, is dynamically saturated in the block " $\mathrm{T}_{\text {RING_LIM }}$ " by the most restrictive and limiting torque between MG/1 (" $\mathrm{T}_{M A X_{-} M G 1}$ ") and ICE (" $\mathrm{T}_{\text {MAX_ICE }}$ "), both of which are converted at the ring. The resulting limited output ring torque reference $T_{R I N G}^{*}$ is transposed at the sun and then applied as reference $T_{M G 1}^{*}$ to the MG/1 driver. The torque reference $T_{M G 1}^{*}$ is applied to $\mathrm{MG} / 1$, taking into account the limitation characteristics and control dynamics of the SPM$\mathrm{SM}$ drive that was used for MG/1. MG/1 output torque $T_{M G 1}$ is then applied to the sun gear of the planetary gear set dynamic model.

\subsubsection{MG/2 Torque Regulation}

The difference between the desired torque $\left(T_{F D}^{*}\right)$, produced by the ground speed regulation loop, and the torque potentially available at the ring ( $\left.T_{R I N G}^{*}\right)$, is defined as missing final drive torque $\left(T_{F D_{\text {missing }}}^{*}\right)$ for the vehicle dynamic control. The $\mathrm{MG} / 2$ machine, directly coupled to the ring, must generate $T_{M G 2}^{*}$, which is obtained as the sum of the dynamic torque $T_{F D_{\text {missing }}}^{*}$ and the torque $T_{P W R_{B L N C}}$, coming from the power $P_{P W R_{B L N C}}$ calculated for the electric power system. With this principle, MG/2 contributes to achieving the power balance of the electric power system. The power $P_{P W R_{B L N C}}$ is obtained as the sum of power $P_{M G 1}$ generated by $M G / 1$ and the additional power $P_{P S}$ requested by the power system (e.g., battery charge/discharge request and/or the connection of additional electric loads).

The torque reference $T_{M G 2}^{*}$ is applied to the MG/2 driver, considering the limit characteristics and control dynamics of the induction machine drive used for MG/2. Algebraic sum $T_{R I N G}$ of $M G / 2$ output torque $T_{M G 2}$ and final drive load torque $T_{L O A D}$ is then applied to the ring gear of the planetary gear set.

\subsubsection{Combustion Engine Speed Regulation}

The ICE base reference speed $\omega_{I C E_{0}}^{*}$ is directly calculated from the vehicle ground speed $V_{G N D}$ using the map given in Section 4.1. Base reference $\omega_{I C E_{0}}^{*}$ is modified by the ICE load regulator, which can increase the ICE base reference speed by the contribution $\Delta \omega_{I C E}^{*}$, obtaining the final ICE speed reference $\omega_{I C E}^{*}$. This speed reference variation ensures a predefined torque margin between the required torque and the maximum torque at the actual speed. In other words, the engine speed can deviate from the base mapped speed to produce the amount of power required by the dynamic transmission model in any operating condition. Final ICE speed reference $\omega_{I C E}^{*}$ is regulated by a speed loop, which calculates the torque reference $T_{I C E}^{*}$ for the ICE engine control unit. $T_{I C E}^{*}$ is computed according to the limit characteristics and control dynamics of the chosen engine. Output engine torque $T_{I C E}$ is transferred to the carrier gear through a step-up gearbox with the gear ratio $\tau_{1}$. The algebraic sum $\left(T_{C A R R I E R}\right)$ of $T_{C_{I C E}}$ PTO, and auxiliary drive load torque $T_{A U X}$, is then applied to the carrier gear of the planetary gear set dynamic model.

To enhance the control system stability, ICE controllers only are calibrated to vary the operating point if the condition requiring this persists for at least a few seconds. Fast transients (such as those caused by the presence of clods or bumps on the ground) are managed by varying the MG/2 torque reference, which eventually uses the battery to meet the power demand until the ICE changes its operating point, as shown in the examples in Figures 8-12. 


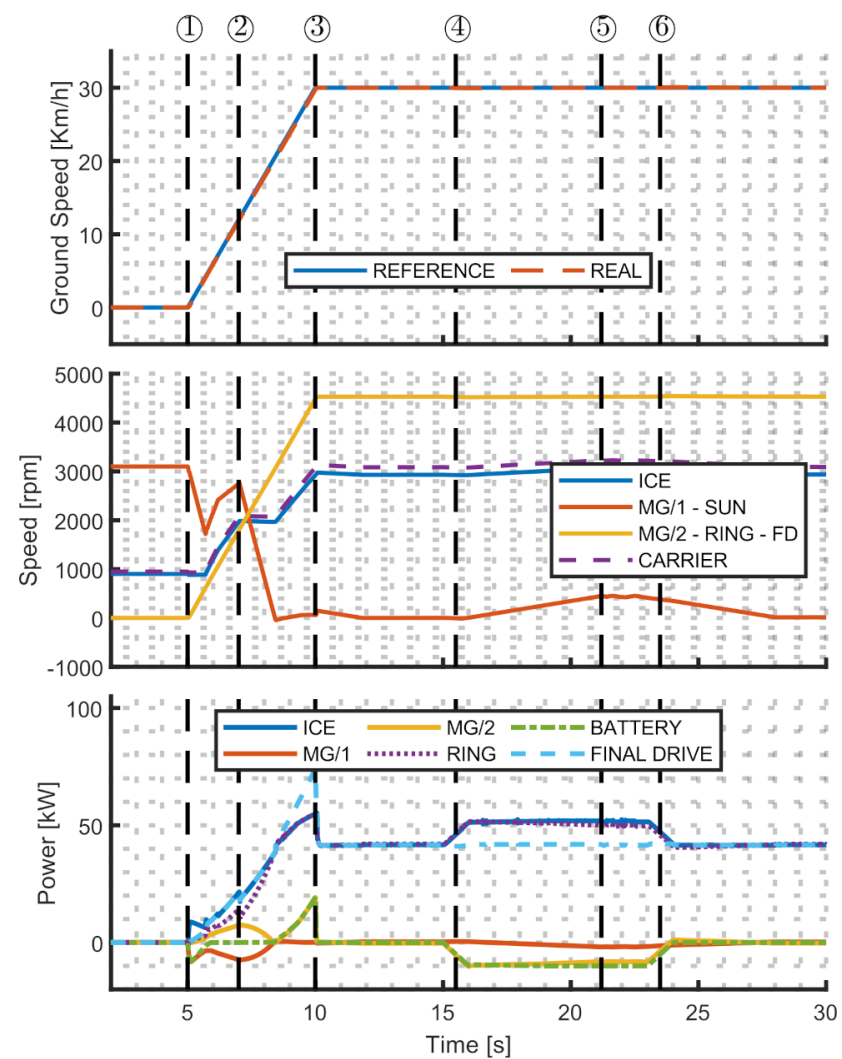

Figure 8. Ground speed (top), drivetrain elements speed (middle), and drivetrain elements power (bottom) during vehicle acceleration, traveling at a constant speed and electric power request.
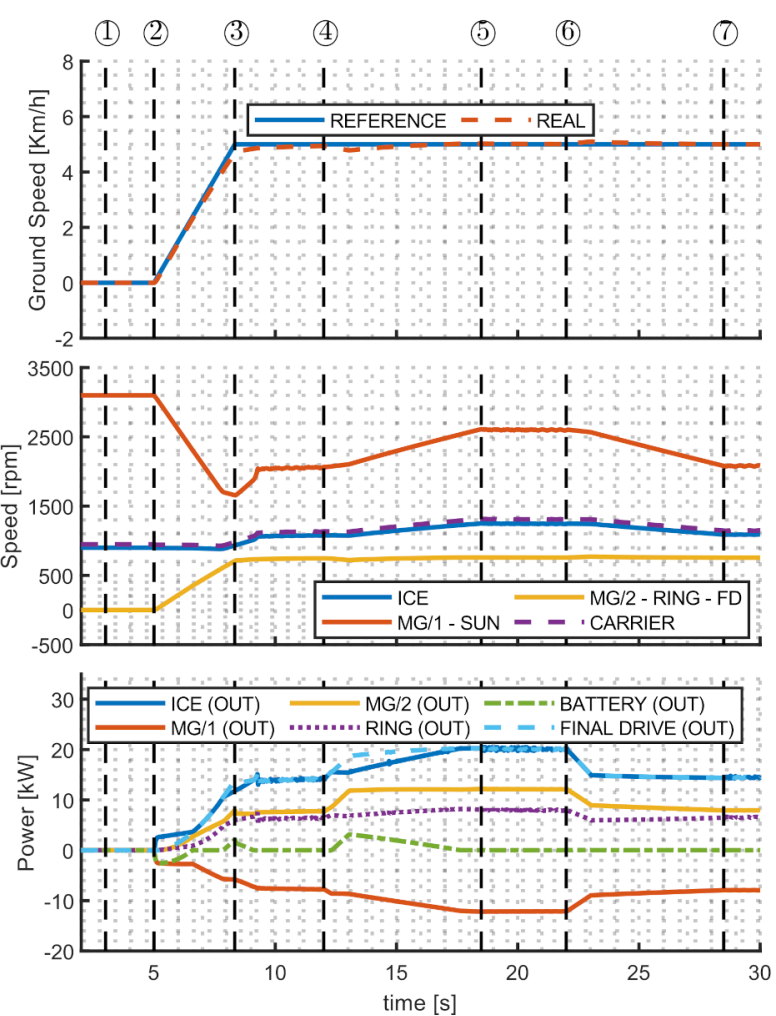

Figure 9. Ground speed (top), drivetrain elements speed (middle), and drivetrain elements power (bottom) during vehicle acceleration, traveling at low speed with the application of heavy load. 

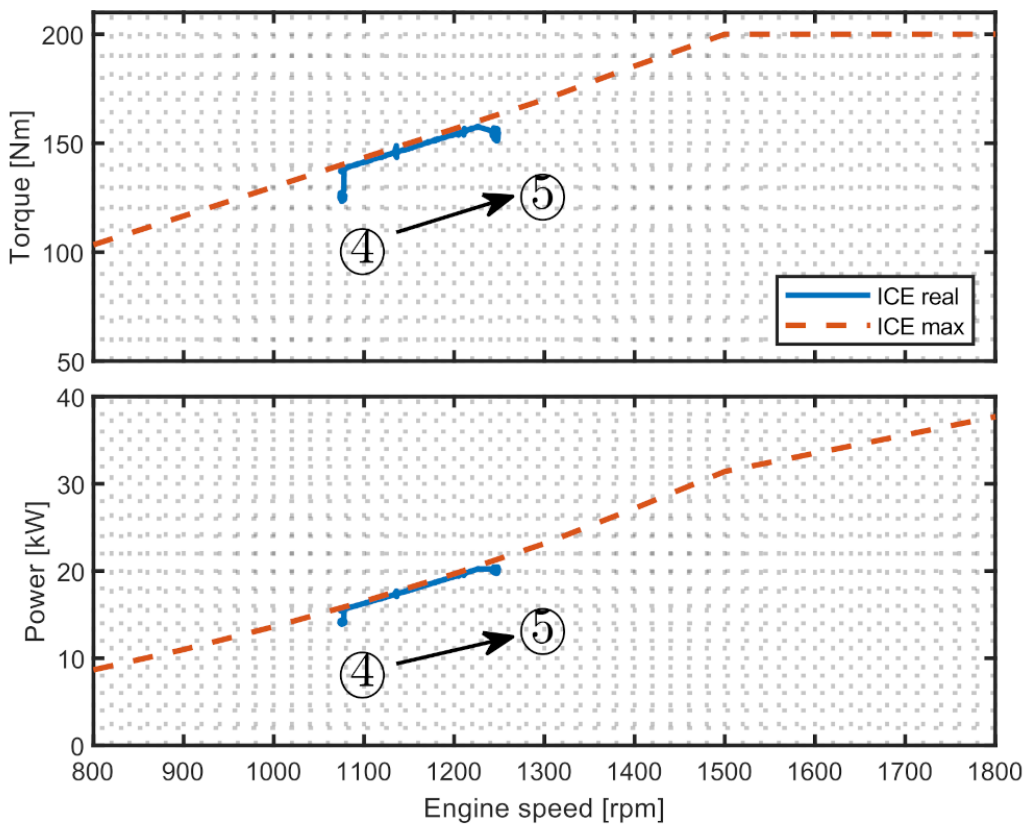

Figure 10. Torque-speed and power-speed operating point modifications of the ICE, due to a step change of the pulling load from $10,000 \mathrm{~N}$ to $14,000 \mathrm{~N}$ at $5 \mathrm{~km} / \mathrm{h}$.
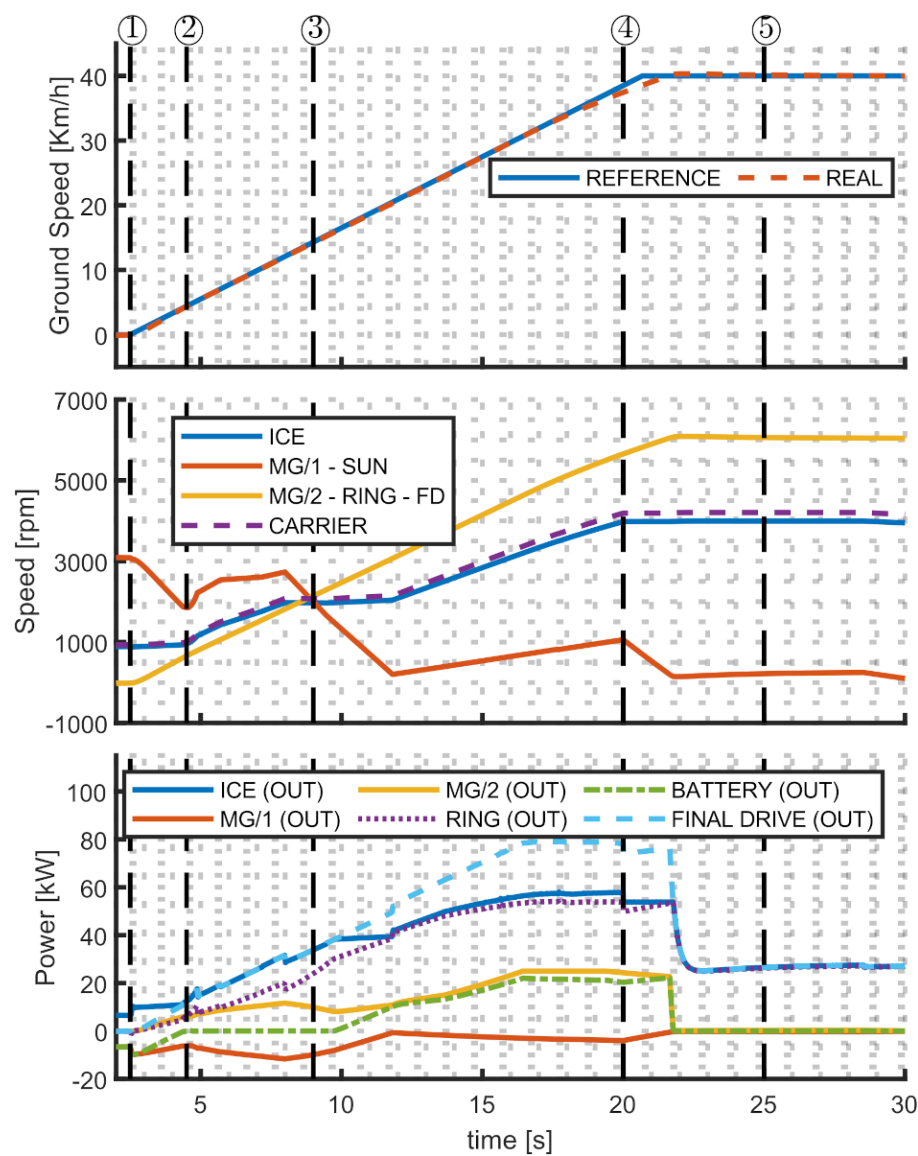

Figure 11. Ground speed (top), drivetrain elements speed (middle), and drivetrain elements power (bottom) during vehicle acceleration, traveling at high speed with the application of heavy load. 

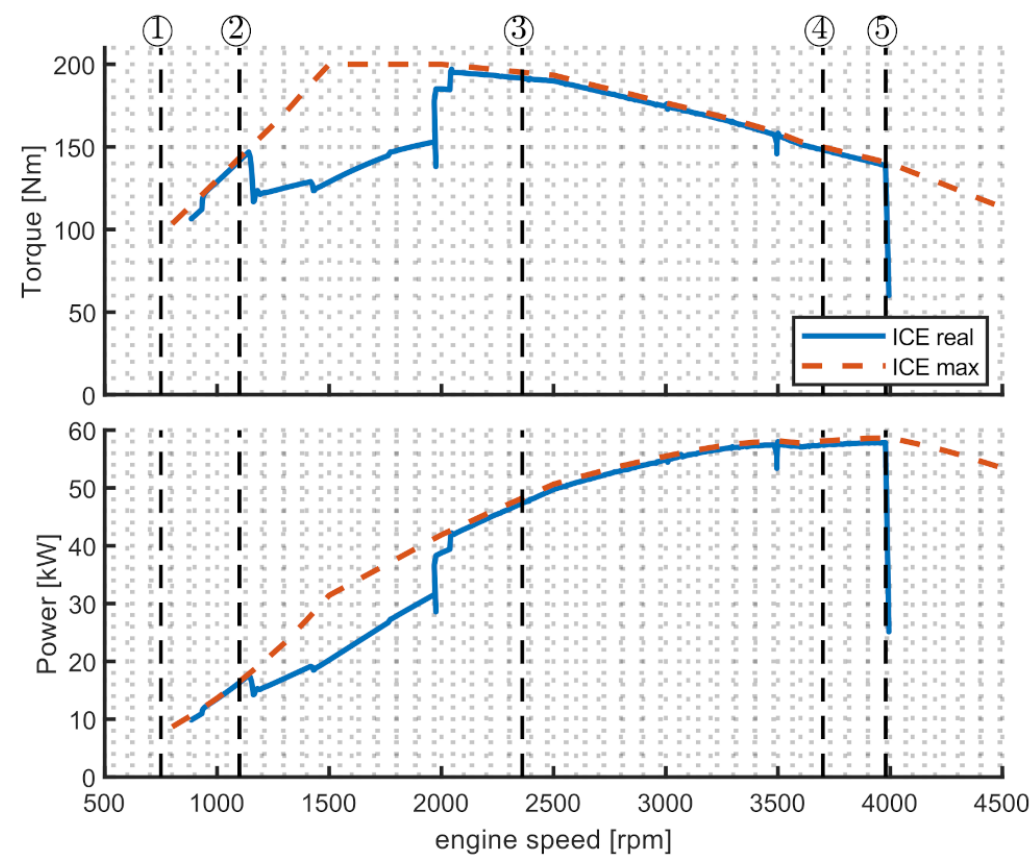

Figure 12. Torque-speed and power-speed operating point modifications of the ICE during the acceleration test at high load.

\section{Numerical Validation and Results}

The control system described in Section 4 has been fully developed in the Matlab/Simulink (MathWorks) environment. The epicyclical gear set model and the vehicle dynamics model have been implemented using the state-space variable representation given in Section 2.2. Simplified standard dynamic models, the limit characteristics of the two electric drives MG/1 and MG/2 [32], and limit characteristics of the combustion engine are used.

Using the control algorithm schematized in Figure 7, several tests have been carried out in a wide variety of operating conditions. The three main tests reported are as follows: acceleration at medium speed while supplying electric power, low-speed operation with a very high pulling force applied to the tractor, and full power acceleration at high speed.

Figure 8 shows the dynamic driveline behavior for an acceleration test. It displays an acceleration from 0 to $30 \mathrm{~km} / \mathrm{h}$ in $5 \mathrm{~s}$ (1)-(2)-(3)), then a constant speed reference was applied (4)-(5)-(6)). At $30 \mathrm{~km} / \mathrm{h}$ (5)), $10 \mathrm{~kW}$ of power were requested as an external electric load, and then this power request was removed (6). A variable pulling force, proportional to the ground speed, with a coefficient of $600 \mathrm{Ns} / \mathrm{m}$ was applied during the whole test. Figure 8 (top) shows that the control algorithm and the system can effectively follow the ground speed reference during acceleration and during travel at a constant speed. A step in electric power demand (from the external power system) can be satisfied without appreciable effects on the actual ground speed. Figure 8 (middle) shows the rotating speed of the main elements of the drivetrain. This behavior reflects the kinematic diagrams of Figure 4 for the engine base reference speed map. In particular, it can be observed that MG/1 can be stopped at high vehicle speed (4) if the driveline does not produce additional power for the electrical power system. Figure 8 (bottom) shows the power flow in the different sections of the driveline. This diagram helps to understand the contribution of the MG/2 drive. During acceleration, MG/2 contributed, starting from zero speed (1), then behaving as a motor, absorbing the power generated by MG/1 (2) with zero power exchanged with the battery. When requested from the vehicle dynamics control, MG/2 added power to the final drive, taking power from both MG/1 and the battery (3)) (fullhybrid mode). In steady-state conditions at $30 \mathrm{~km} / \mathrm{h}$ (4)), if no power must be exchanged with the external power system, MG/2 does not operate (combustion-only mode). The 
electric power request between $15 \mathrm{~s}$ and $23 \mathrm{~s}$ (4)-(5)-(6) is satisfied by operating MG/2 as a generator, allowing constant output power. The role of $\mathrm{MG} / 1$ can also be analyzed in the diagram of Figure 8. During acceleration (1)-(2)), MG/1 is controlled to increase the ICE load; thus, it operates as a generator. All the power produced by MG/1 can be depleted by $M G / 2$, except during the initial transient when $M G / 2$ speed is around zero (1). At medium-high speed (3)-(4)), with MG/1 stopped in a steady-state condition, it does not exchange power.

Figures 9 and 10 show the dynamic behavior of the driveline for an acceleration test. It displays an acceleration from 0 to $5 \mathrm{~km} / \mathrm{h}$ in $3.5 \mathrm{~s}$ under a heavy load (2)-(3). The load was a variable pulling force proportional to ground speed, with a rate of $7500 \mathrm{Ns} / \mathrm{m}$, corresponding to about $10,000 \mathrm{~N}$ at $5 \mathrm{~km} / \mathrm{h}$. Constant ground speed reference is given in (3)-(4)-(5)-(6)-(7). At this speed, an additional pulling torque of $4000 \mathrm{~N}$ was applied (4)), kept constant for a few seconds (4)-(5)-(6) and then removed (6). The scope of the test was to verify the capability of the driveline regulation system to change the combustion engine operating point from the base reference speed $\omega_{I C E_{0}}^{*}$, for satisfying the system's overall power demand. Figure 9 (top) shows that the drivetrain effectively follows the ground speed reference during acceleration and constant speed traveling. The load step at (4) resulted in a minimal ground speed variation. Figure 9 (middle) shows the rotating speed of the main elements of the drivetrain. In particular, it can be observed that the MG/1 speed increased when the additional load was applied (4)-(5)-(6)). This MG/1 acceleration was due to increased engine speed, from the pre-mapped base reference to the new point, with a higher power. Figure 9 (bottom) shows the power flow in the different sections of the driveline. During acceleration from zero speed to $5 \mathrm{~km} / \mathrm{h}$, the system's behavior was similar to the previous case, with MG/1 loading the ICE, generating power that MG/2 uses (2)-(3)). The load change (4) determined a sudden increase in MG/2 power for faster response to the load demand. The new power demand could not be satisfied by the ICE operating at the pre-mapped reference speed $\left(\omega_{I C E_{0}}^{*}=1080 \mathrm{rpm}\right)$ but only at the new increased speed $\left(\omega_{I C E}=1250 \mathrm{rpm}\right)$. During the engine transient (4)-(5)), the additional required power was supplied by the battery pack. As the new engine operating point was reached, MG/2 no longer drained power from the battery (5)). The variation of the engine operating point from the pre-mapped base reference speed $\left(\omega_{I C E_{0}}^{*}\right)$ in (4), to the new point (5), is shown on the speed vs. torque diagram and the speed vs. power diagram of the engine, given in Figure 10.

Figure 11 shows the dynamic behavior of the driveline for an acceleration test. It displays an acceleration from 0 to $40 \mathrm{~km} / \mathrm{h}$ in $20 \mathrm{~s}$, under a heavy load (representing a trailer of 5 tons) pulled on a flat road. This acceleration test required very high power, which was higher than the engine's maximum power.

By analyzing the diagram of Figure 11 (bottom), it can be seen that the first acceleration interval (2)-(3) was carried out without exchanging power with the batteries. Conversely, in the second interval (3)-(4)), the battery delivered additional power for supplying MG/2. In this way, a peak power of $80 \mathrm{~kW}$ can be reached at the final tractor driveline during the acceleration transient. The variation of the engine's operating point, from the pre-mapped speed during acceleration, is more clearly shown in Figure 12. This figure shows that the engine was fully loaded during the acceleration, reaching peak power of $58 \mathrm{~kW}$ at $4000 \mathrm{rpm}$.

\section{Tractor Prototype}

The proposed solution has been developed as a full-scale vehicle prototype. The transmission layout is based on the proposed coaxial and concentric arrangement of the MG/1 and MG/2 machines, which are fully integrated with the planetary gear set. All the data of the vehicle, engine transmission, and drives correspond to those given in Appendix A.

A picture of the experimental tractor is given in Figure 13. This figure shows the core of the transmission corresponding to the e-CVT layout from Figure 2, which is located at 
the center of the tractor, under the driver seat. Some important parts of the transmission are also shown in the picture; in particular, the combustion engine, the cooling box, the carrier shaft, one inverter, and additional mechanical loads constituted of hydraulic pumps. The e-CVT transmission is coupled, on the rear side of the tractor, with a standard tractor final driveline. The battery pack is safely installed in a box on the roof of the cabin.
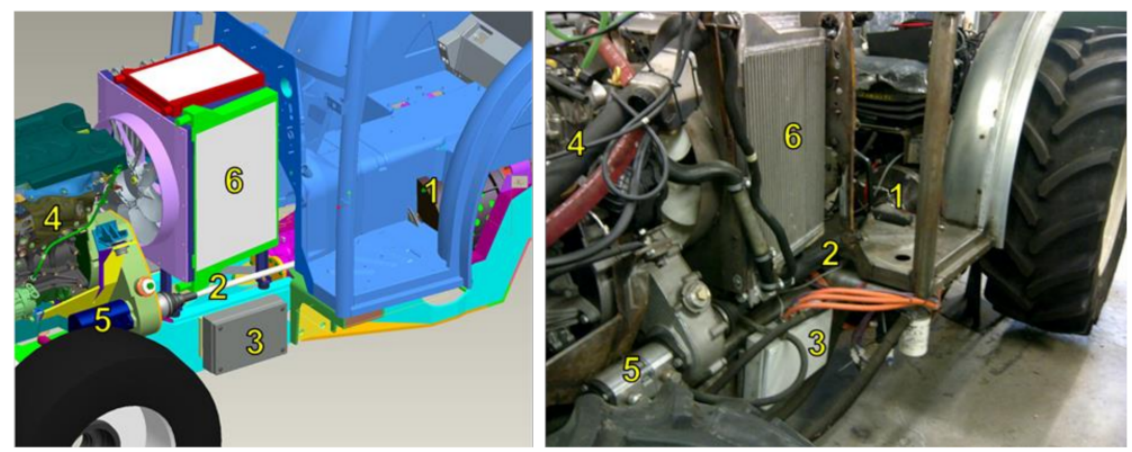

Figure 13. CAD design (left) and actual picture (right) of the experimental setup. 1-e-CVT; 2-carrier shaft; 3-MG/2 driver; 4-ICE; 5-ancillaries (hydraulic pump); 6-cooling box.

Some additional features of the prototype can be appreciated from the picture: the flat cabin floor, the cooling box arrangement constituted by parallel radiators, and the advanced position of the engine with respect to the cabin. All these features-which are made possible by the reduced size and mechanical layout of the coaxial and concentric eCVT solution-allow the engine to be moved away from the driver, with double advantages of improving comfort and improving the weight distribution of the front axle.

The control architecture of the vehicle's prototype has been fully implemented on National Instruments' Compact RIO (NI cRIO) real-time controller. This device is equipped with analog and digital inputs for managing operator controls, high power digital outputs to control relays and valves, and four CAN interfaces-which allow communication with the engine, the electric drives, the battery management system, and the HMI. The "lowlevel" control architecture (mainly the I/O management and a few signal-conditioning algorithms) are implemented on the cRIO-FPGA controller. Moreover, the whole powersplit e-CTV driveline control architecture, described in Section 4, has been fully developed and tested in Matlab/Simulink (Mathworks) and then compiled and executed directly on the NI cRIO real-time processor that is installed on the prototype.

This experimental vehicle has the fundamental scope to prove the technical feasibility of a hybrid electric drivetrain for tractors; therefore, primarily demonstrating that the volume and weight distribution of the proposed power-split e-CVT complies with standard small-size tractor requirements. This prototype has the potential to demonstrate the functionality and the performance of such a transmission concept, in a plurality of operating conditions.

Future experimental verification will provide a more detailed comparison between standard agricultural tractors and the presented architecture, concerning specifics such as fuel economy capability, multimodal efficiency improvement, noise, vibrations, and harshness (NVH) reduction, while considering a multitude of energy management systems [33]. The type of battery pack (e.g., $\mathrm{LiFePO}_{4}$, Li-ion, or Supercapacitor aided) will play a vital role in determining whether the energy management strategy will be online, offline, learning-based, predictive, genetic, dynamic, or convex, etc. [34-39].

\section{Conclusions}

A solution for full-hybrid transmission is investigated in this paper for application in agricultural tractors. The main possible operating modes are hybrid mode, full-electric, combustion-only, and parallel-hybrid. The paper mainly focuses on selecting and designing 
electro-mechanical components and developing a streamlined transmission control strategy for the hybrid mode operation.

The full model of the vehicle, the transmission, and the control strategy have been developed to numerically demonstrate the capability of the proposed power-split e-CVT to drive the tractor under even the most severe conditions that can be present during the use of an agricultural tractor. The basic vehicle performances that are obtained by the e-CVT are comparable with those of standard agricultural tractors with combustion engines and mechanical transmissions. This paper also demonstrates the availability of additional, important features embedded in the proposed power-split e-CVT, such as power boost, independent PTO control, and the capability of supplying electric power. The study also led to the realization of a prototype agricultural tractor for use in orchards. This has made it possible to demonstrate the feasibility of accommodating all the hybrid powertrain components in a small-sized specialized tractor. It is also shown that new possibilities for the positioning of the main components could improve the weight distribution and the comfort of the driver. The prototype will allow future experimental verification, which will provide information on the actual performance of the proposed solution and make it possible to compare the performance of the presented architecture with conventional systems.

Significant research should follow this proposal, to analyze all the functional modes not yet verified (full-electric, parallel-hybrid, and PTO-combined control, etc.) and evaluate fuel economy with respect to more traditional drivelines. A relevant aspect that should be investigated in upcoming research is the energy management system of this hybrid driveline, which could be addressed using either standard approaches as those given in [34] and [40], or online learning-based strategies [41]. These energy control algorithms will be optimized in terms of fuel consumption, depending on the considered battery pack and its storage technology. Besides, the proposed powertrain will also be tested on vehicle-specific working cycles such as the ISO 8178 non-road steady cycle and the non-road transient cycle $[42,43]$. Eventually, more detailed models of the vehicle's components such as the battery [44,45], the driveline parts [46], and the ancillaries [47] will be introduced.

Considering the recent developments of battery technologies suitable for plug-in hybrid electric vehicles (PHEV) that are spreading in the automotive sector, this paper opens a new perspective for hybrid power-split e-CVT research, in the unexplored sector of agricultural tractors.

Author Contributions: Conceptualization, C.R. and D.P.; methodology, C.R. and D.P.; software, D.P., C.F. and M.B.; validation, C.R., D.P. and R.M.; formal analysis, C.R. and D.P.; investigation, C.R., D.P., C.F., M.B. and R.M.; resources, C.R., D.P. and R.M.; data curation, D.P., C.F. and M.B.; writing —original draft preparation, C.R., D.P., C.F., M.B. and R.M.; writing—review and editing, C.R., D.P., C.F., M.B. and R.M.; visualization, D.P. and R.M.; supervision, C.R., D.P. and R.M. All authors have read and agreed to the published version of the manuscript.

Funding: This research received no external funding.

Institutional Review Board Statement: Not applicable.

Informed Consent Statement: Not applicable.

Data Availability Statement: Data is available in the paper.

Conflicts of Interest: The authors declare no conflict of interest. 


\section{Appendix A}

Table A1. Vehicle main data and requirements. Data adapted from [29].

\begin{tabular}{ccc}
\hline Data & Value & Unit \\
\hline Gross weight & 2500 & $\mathrm{~kg}$ \\
Size $\mathrm{L} \times \mathrm{W} \times \mathrm{H}$ & $3 \times 2 \times 2.5$ & $\mathrm{~m}$ \\
Front wheel radius & 0.425 & $\mathrm{~m}$ \\
Rear wheel radius & 0.650 & $\mathrm{~m}$ \\
Max speed & 40 & $\mathrm{~km} / \mathrm{h}$ \\
Max pull force at $0 \mathrm{~km} / \mathrm{h}$ & 22,000 & $\mathrm{~N}$ \\
Power at max speed & 80 & $\mathrm{~kW}$ \\
Plowing speed & 10 & $\mathrm{~km} / \mathrm{h}$ \\
Plowing power & 40 & $\mathrm{~kW}$ \\
Max auxiliary power & 6 & $\mathrm{~kW}$ \\
\hline
\end{tabular}

Table A2. Thermal engine data. Data adapted from [29].

\begin{tabular}{ccc}
\hline Data & Value & Unit \\
\hline Type & \multicolumn{2}{c}{ Diesel engine for cars } \\
Common rail injection & 1600 & $\mathrm{bar}$ \\
Displacement & 1298 & $\mathrm{~cm}^{3}$ \\
Max power & 59 & $\mathrm{~kW}$ \\
Speed at max power & 4000 & $\mathrm{rpm}$ \\
Max torque & 200 & $\mathrm{Nm}$ \\
Speed at max torque & $1500-2000$ & $\mathrm{rpm}$ \\
\hline
\end{tabular}

Table A3. Driveline gear ratios. Data adapted from [29].

\begin{tabular}{cccc}
\hline Data & & Value & Unit \\
\hline Epicyclical gear ratio & $\tau_{0}$ & -2.1915 & - \\
Engine to carrier gear ratio & $\tau_{1}$ & 0.9500 & - \\
Eng. to PTO gear ratio 540 & $\tau_{2}$ & 0.2224 & - \\
Eng. to PTO gear ratio 1000 & $\tau_{3}$ & 0.3804 & - \\
Engine to pumps gear ratio & $\tau_{4}$ & 0.9500 & - \\
Final drive gear ratio (rear) & $\tau_{5}$ & 0.0270 & - \\
Final drive gear ratio (front) & $\tau_{6}$ & 0.0430 & $\mathrm{~kg}$ \\
e-CVT main assembly weight & 80 & $\mathrm{~mm}$ \\
e-CVT main assembly diameter & 320 & $\mathrm{~mm}$ \\
e-CVT main assembly length & 350 & \\
\hline
\end{tabular}

Table A4. Electric drives and storage. Data adapted from [29].

\begin{tabular}{cccc}
\hline Element & Data & Value & Unit \\
\hline & Machine type & \multicolumn{2}{c}{ PMSM-SM $^{1}$} \\
MG/1 rated torque & 70 & $\mathrm{Nm}$ \\
MG/1 & MG/1 rated speed & 3000 & $\mathrm{Rpm}$ \\
& MG/1 max. speed & 5700 & $\mathrm{Rpm}$ \\
& Machine type & 22 & $\mathrm{~kW}$ \\
\hline & Maximum torque & 250 & $\mathrm{IM}^{2}$ \\
& Speed @ maximum torque & 1500 & $\mathrm{Nm}$ \\
MG/2 & Rated torque & 100 & $\mathrm{rpm}$ \\
& Rated speed & 2500 & $\mathrm{Nm}$ \\
& Maximum speed & 7000 & $\mathrm{rpm}$ \\
& Rated voltage & 140 & $\mathrm{rpm}$ \\
\hline DC-link & Rated energy & 15 & $\mathrm{~V}$ \\
\hline \multirow{2}{*}{ Battery } & Type & $\mathrm{kWh}$ \\
& Li-Po high-power \\
\hline
\end{tabular}

${ }^{1}$ Surface Permanent Magnet-Synchronous Machine (SMPM-SM). ${ }^{2}$ Induction Motor (IM). 


\section{References}

1. Sasaki, S. Toyota's newly developed hybrid powertrain. In Proceedings of the 10th International Symposium on Power Semiconductor Devices and ICs, ISPSD’98, Kyoto, Japan, 3-6 June 1998; pp. 17-22.

2. Dagci, O.H.; Peng, H.; Grizzle, J.W. Hybrid electric powertrain design methodology with planetary gear sets for performance and fuel economy. IEEE Access 2018, 6, 9585-9602. [CrossRef]

3. Miller, J.M.; Schulz, S.E.; Conlon, B.; Duvall, M.; Kankam, M.D.; Nagel, N. Adjustable Speed Drives Transportation Industry Needs Part I: Automotive. In Proceedings of the 2003 IEEE 58th Vehicular Technology Conference, VTC 2003-Fall, Orlando, FL, USA, 6-9 October 2003; Volume 5, pp. 3220-3225.

4. Yimin, G.; Ehsani, M.; Miller, J.M. Hybrid Electric Vehicle: Overview and State of the Art. In Proceedings of the IEEE International Symposium on Industrial Electronics, ISIE 2005, Dubrovnik, Croatia, 20-23 June 2005; pp. 307-316.

5. Korlath, G. Mobility analysis of off-road vehicles: Benefits for development, procurement and operation. J. Terramech. 2007, 44, 383-393. [CrossRef]

6. Gupta, S.; Maity Sr, R.; Kulkarni Ceng, S. Modeling, Analysis and Experimental Validation of Tractor Architectures for Rural Electrification. In Proceedings of the 8th SAEINDIA International Mobility Conference \& Exposition and Commercial Vehicle Engineering Congress 2013 (SIMCOMVEC), Chennai, India, 4-7 December 2013.

7. El Bassam, N.; Maegaard, P. Integrated Renewable Energy for Rural Communities; Elsevier: Amsterdam, The Netherlands, 2004; ISBN 9780444510143.

8. Scolaro, E.; Alberti, L.; Barater, D. Electric Drives for Hybrid Electric Agricultural Tractors. In Proceedings of the 2021 IEEE Workshop on Electrical Machines Design, Control and Diagnosis (WEMDCD), Modena, Italy, 8-9 April 2021; pp. 331-336.

9. Troncon, D.; Alberti, L.; Mattetti, M. A Feasibility Study for Agriculture Tractors Electrification: Duty Cycles Simulation and Consumption Comparison. In Proceedings of the 2019 IEEE Transportation Electrification Conference and Expo (ITEC), Detroit, MI, USA, 19-21 June 2019.

10. Moreda, G.P.; Muñoz-García, M.A.; Barreiro, P. High voltage electrification of tractor and agricultural machinery-A review. Energy Convers. Manag. 2016, 115, 117-131. [CrossRef]

11. Bouquain, D.; Blunier, B.; Miraoui, A. HEV series architectures evaluation: Modeling, simulation and experimentation. In Proceedings of the 2009 IEEE Vehicle Power and Propulsion Conference, Dearborn, MI, USA, 7-10 September 2009; pp. 584-591.

12. Abul Masrur, M. Hybrid and Electric Vehicle (HEV/EV) Technologies for Off-Road Applications. Proc. IEEE 2021, 109, 1077-1093. [CrossRef]

13. Renken, F.; Wolf, J. Power electronics for hybrid-drive systems. In Proceedings of the 2007 European Conference on Power Electronics and Applications, Aalborg, Denmark, 2-5 September 2007; pp. 1-10.

14. Yang, Y.; Ali, K.A.; Roeleveld, J.; Emadi, A. State-of-the-art electrified powertrains-hybrid, plug-in, and electric vehicles. Int. J. Powertrains 2016, 5, 1. [CrossRef]

15. Mocera, F.; Somà, A. Analysis of a Parallel Hybrid Electric Tractor for Agricultural Applications. Energies 2020, 13, 3055. [CrossRef]

16. Zhao, D.; Stobart, R.; Dong, G.; Winward, E. Real-Time Energy Management for Diesel Heavy Duty Hybrid Electric Vehicles. IEEE Trans. Control Syst. Technol. 2015, 23, 829-841. [CrossRef]

17. Dalboni, M.; Santarelli, P.; Patroncini, P.; Soldati, A.; Concari, C.; Lusignani, D. Electrification of a Compact Agricultural Tractor: A Successful Case Study. In Proceedings of the 2019 IEEE Transportation Electrification Conference and Expo (ITEC), Detroit, MI, USA, 19-21 June 2019.

18. Hefel, K. Electric Transmission Gearing. U.S. Patent US2223210A, 20 January 1935.

19. Holmes, A.G.; Schmidt, R.M. Hybrid Electric Powertrain Including a Two-Mode Electrically Variable Transmission. U.S. Patent US6478705B1, 12 November 2002.

20. Miller, J.M. Hybrid electric vehicle propulsion system architectures of the e-CVT type. IEEE Trans. Power Electron. 2006, 21, 756-767. [CrossRef]

21. Ai, X.; Mohr, T.; Anderson, S. An Electro-Mechanical Infinitely Variable Speed Transmission. In Proceedings of the SAE 2004 World Congress \& Exhibition, Detroit, MI, USA, 8-11 March 2004.

22. Villeneuve, A. Dual Mode Electric Infinitely Variable Transmission. In SAE Technical Papers; SAE International: Warrendale, PA, USA, 2004.

23. Grewe, T.M.; Conlon, B.M.; Holmes, A.G. Defining the General Motors 2-Mode Hybrid Transmission. In Proceedings of the SAE World Congress \& Exhibition, Detroit, MI, USA, 16-19 April 2007.

24. Muta, K.; Yamazaki, M.; Tokieda, J. Development of New-Generation Hybrid System THS II-Drastic Improvement of Power Performance and Fuel Economy. In Proceedings of the SAE 2004 World Congress \& Exhibition, Dearborn, MI, USA, 8-11 March 2004.

25. Kimura, A.; Ando, I.; Itagaki, K. Development of Hybrid System for SUV. In Proceedings of the SAE 2005 World Congress \& Exhibition, Detroit, MI, USA, 11-14 April 2005.

26. Schulz, M. Circulating mechanical power in a power-split hybrid electric vehicle transmission. Proc. Inst. Mech. Eng. Part D J. Automob. Eng. 2004, 218, 1419-1425. [CrossRef]

27. Yamaguchi, J. Project G21 to Prius. SAE Automot. Eng. Int. 2004, 42-44.

28. Passenger Cars I European Alternative Fuels Observatory-EAFO. Available online: https://www.eafo.eu/vehicles-and-fleet/m1 (accessed on 6 July 2020). 
29. Rossi, C.; Pontara, D.; Casadei, D. e-CVT Power Split Transmission for Off-Road Hybrid-Electric Vehicles. In Proceedings of the 2014 IEEE Vehicle Power and Propulsion Conference (VPPC), Coimbra, Portugal, 27-30 October 2014; pp. 1-6.

30. Rossi, C. e-CVT Power Split Transmission for Hybrid-Electric Vehicles. In Proceedings of the 2013 IEEE Vehicle Power and Propulsion Conference (VPPC), Beijing, China, 15-18 October 2013; pp. 1-8.

31. Yu, Y.; Gao, Y.; Peng, H.; Wang, Q. Parametric design of power-split HEV drive train. In Proceedings of the 2009 IEEE Vehicle Power and Propulsion Conference, Dearborn, MI, USA, 7-10 September 2009; pp. 1058-1063.

32. Lukic, S.M.; Emado, A. Modeling of electric machines for automotive applications using efficiency maps. In Proceedings of the Electrical Insulation Conference and Electrical Manufacturing and Coil Winding Technology Conference, Indianapolis, IN, USA, 25 September 2003; pp. 543-550.

33. Qin, Y.; Tang, X.; Jia, T.; Duan, Z.; Zhang, J.; Li, Y.; Zheng, L. Noise and vibration suppression in hybrid electric vehicles: State of the art and challenges. Renew. Sustain. Energy Rev. 2020, 124, 109782. [CrossRef]

34. Zhang, F.; Wang, L.; Coskun, S.; Pang, H.; Cui, Y.; Xi, J. Energy Management Strategies for Hybrid Electric Vehicles: Review, Classification, Comparison, and Outlook. Energies 2020, 13, 3352. [CrossRef]

35. Masih-Tehrani, M.; Ebrahimi-Nejad, S. Hybrid Genetic Algorithm and Linear Programming for Bulldozer Emissions and FuelConsumption Management Using Continuously Variable Transmission. J. Constr. Eng. Manag. 2018, 144, 04018053. [CrossRef]

36. Buccoliero, G.; Anselma, P.G.; Bonab, S.A.; Belingardi, G.; Emadi, A. A New Energy Management Strategy for Multimode Power-Split Hybrid Electric Vehicles. IEEE Trans. Veh. Technol. 2020, 69, 172-181. [CrossRef]

37. Shi, D.; Wang, S.; Cai, Y.; Chen, L. Stochastic Predictive Energy Management of Power Split Hybrid Electric Bus for Real-World Driving Cycles. IEEE Access 2018, 6, 61700-61713. [CrossRef]

38. Eldeeb, H.H.; Elsayed, A.T.; Lashway, C.R.; Mohammed, O. Hybrid Energy Storage Sizing and Power Splitting Optimization for Plug-In Electric Vehicles. IEEE Trans. Ind. Appl. 2019, 55, 2252-2262. [CrossRef]

39. Zeng, X.; Cui, C.; Wang, Y.; Li, G.; Song, D. Segemented Driving Cycle Based Optimization of Control Parameters for Power-Split Hybrid Electric Vehicle with Ultracapacitors. IEEE Access 2019, 7, 90666-90677. [CrossRef]

40. He, X.; Parten, M.; Maxwell, T. Energy Management Strategies for a Hybrid Electric Vehicle. In Proceedings of the 2005 IEEE Vehicle Power and Propulsion Conference, Chicago, IL, USA, 7 September 2005; pp. 536-540.

41. Chen, J.-S.; Salman, M. Learning Energy Management Strategy for Hybrid Electric Vehicles. In Proceedings of the 2005 IEEE Vehicle Power and Propulsion Conference, Chicago, IL, USA, 7 September 2005; pp. 68-73.

42. ISO 8178-4:2020—Reciprocating Internal Combustion Engines—Exhaust Emission Measurement—Part 4: Steady-State and Transient Test Cycles for Different Engine Applications. Available online: https:/ / www.iso.org/standard/79199.html (accessed on 12 October 2021).

43. Wimalendra, R.S.; Udawatta, L.; Edirisinghe, E.M.C.P.; Karunarathna, S. Determination of Maximum Possible Fuel Economy of HEV for Known Drive Cycle: Genetic Algorithm Based Approach. In Proceedings of the 2008 4th International Conference on Information and Automation for Sustainability, Colombo, Sri Lanka, 12-14 December 2008; pp. 289-294.

44. He, X. Battery Modeling for HEV Simulation Model Development. In Proceedings of the SAE 2001 World Congress, Detroit, MI, USA, 1 February 2001.

45. Miller, J.M.; McCleer, P.J.; Everett, M. Comparative assessment of ultra-capacitors and advanced battery energy storage systems in PowerSplit electronic-CVT vehicle powertrains. In Proceedings of the IEEE International Conference on Electric Machines and Drives, 2005, San Antonio, TX, USA, 15 May 2005; pp. 1513-1520.

46. Chung, C.-T.; Wu, C.-H.; Hung, Y.-H. A design methodology for selecting energy-efficient compound split e-CVT hybrid systems with planetary gearsets based on electric circulation. Energy 2021, 230, 120732. [CrossRef]

47. Syed, F.U.; Kuang, M.L.; Czubay, J.; Ying, H. Derivation and Experimental Validation of a Power-Split Hybrid Electric Vehicle Model. IEEE Trans. Veh. Technol. 2006, 55, 1731-1747. [CrossRef] 